\title{
EFEITOS ESPECIAIS EM COMPUTAÇÃO GRÁFICA - MORPHING
}

Breno Malacrida dos Santos ${ }^{1}$, Almir Olivette Artero $^{2}$

${ }^{1}$ FIPP - Faculdade de Informática - Universidade do Oeste Paulista-UNOESTE. ${ }^{2}$ FCT - Faculdade de Ciências e Tecnologia Universidade Estadual Paulista-UNESP. Email: almir@fct.unesp.br

\section{RESUMO}

Os Efeitos Especiais têm sido muito utilizados na televisão e no cinema, permitindo a realização de cenas impossíveis de serem feitas usando atores reais, devido à causa de grandes riscos à integridade física ou por causa de outras impossibilidades. Este artigo apresenta um aplicativo que que realiza a implementação de efeitos especiais, focando principalmente o efeito de Morphing, possibilitando a sua continuação em trabalhos de simulação.

Palavras-chave: Computação Gráfica, Morphing, Efeitos Especiais.

\section{SPECIAL EFFECTS IN COMPUTER GRAPHICS - MORPHING}

\section{ABSTRACT}

The special effects have long been used in television and movies, enabling the creation of scenes impossible to be made using real actors, because the cause of major risks to the physical or because of other impossibilities. This paper presents an application that performs the deployment of special effects, focusing mainly the effect of morphing, allowing its continued work in simulation.

Keywords: Computer Graphics, Morphing, Special Effects. 


\section{INTRODUÇÃO}

Os Efeitos Especiais têm sido muito utilizados na televisão e no cinema, permitindo a realização de cenas impossíveis de serem feitas usando atores reais, devido à causa de grandes riscos à integridade física ou por causa de outras impossibilidades. A Figura 1 apresenta um efeito sendo usado em uma campanha publicitária.
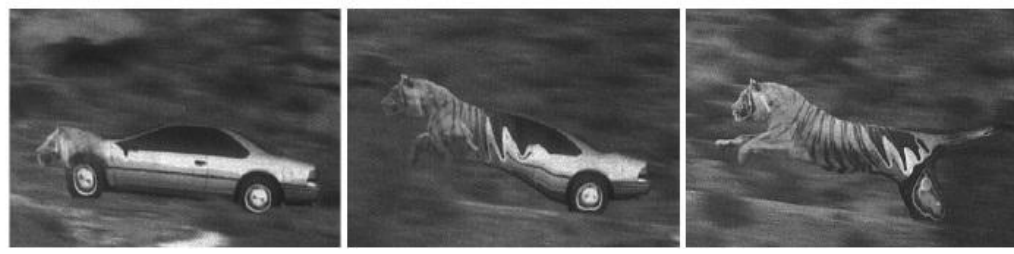

Figura 1. Transformação do automóvel em um tigre.

Porém, tais efeitos também são úteis durante a simulação de experiências em computador, por exemplo, analisando a deformação de objetos submetidos a esforços mecânicos, térmicos, etc. Uma outra aplicação importante é na descrição de pessoas desaparecidas, pois, a partir de uma fotografia antiga da pessoa, é possível estimar as transformações que o tempo pode ter causado em suas feições, tais como em fugitivos da polícia que não possuem fotos atualizadas e sim somente de quando eram mais novos. A implementação destes efeitos implica obrigatoriamente no uso de algum programa de computador, pois a sua implementação manual é praticamente impossível e depende de grande quantidade de cálculos e da necessidade de muitas imagens, pois há a necessidade de ter um sistema computacional que mostre como as pessoas serão em futuro.

\section{TRABALHOS RELACIONADOS}

Nesta seção serão apresentados os recursos utilizados para a criação do aplicativo mediante os problemas encontrados durante sua implementação. Os trabalhos relacionados que foram utilizados durante a implementação do projeto são: Dissolve, Warping, Morphing e a Triangulação.

\section{Morphing}

Define-se Morphing (Gomes e Velho 1994) como um tipo de transformação de duas ou mais imagens envolvendo deformação (Warping) espacial e transformação de tonalidades (Dissolve), obtendo assim uma imagem final derivada de uma transformação gradualmente a partir de uma imagem inicial. Uma das principais utilizações do Morphing é na área de entretenimento, como pode ser visto em diversas produções de cinema, jogos. A Figura 2 ilustra o comercial da Rede de Postos de Combustível, Esso, onde um carro se transforma em um tigre.

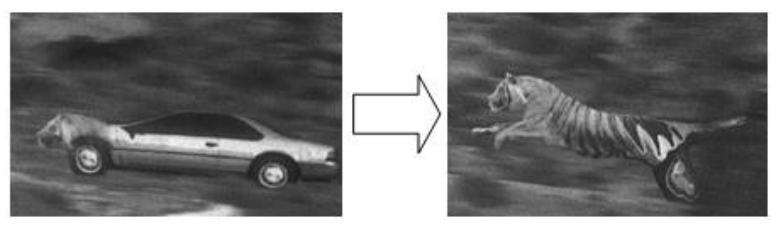

Figura 2. Exemplo de Morphing2.2 Dissolve.

\section{Dissolve}

O Dissolve (Figura 3) é a combinação de cores entre duas imagens, diminuindo a contribuição de cor da primeira e aumentando a contribuição da segunda, através de sequências de interpolações lineares do espaço de cor, aonde temos um conjunto de transformações que depende da variável t, como na Equação 1.

$$
h_{t}(x, y)=(1-t) \cdot f(x, y)+t \cdot g(x, y)
$$


sendo que para $t=0$, têm-se a imagem original $h_{0}(x, y)=f(x, y)$, e para $t=1$, temos a imagem final $h_{1}(x, y)=g(x, y)$. O pseudo-código fica:

$$
\begin{aligned}
& \text { for }(i=0 ; i<=1 ; i+=0.01) \\
& \quad \text { for }(x=0 ; x<=\text { largura; } x++) \\
& \quad \text { for }(y=0 ; y<=\text { altura; } y++) \\
& \quad h(x, y)=f(x, y)^{*}(1-i)+g(x, y){ }^{*} i ;
\end{aligned}
$$

Caixa 1. Pseudo-código para o Dissolve.

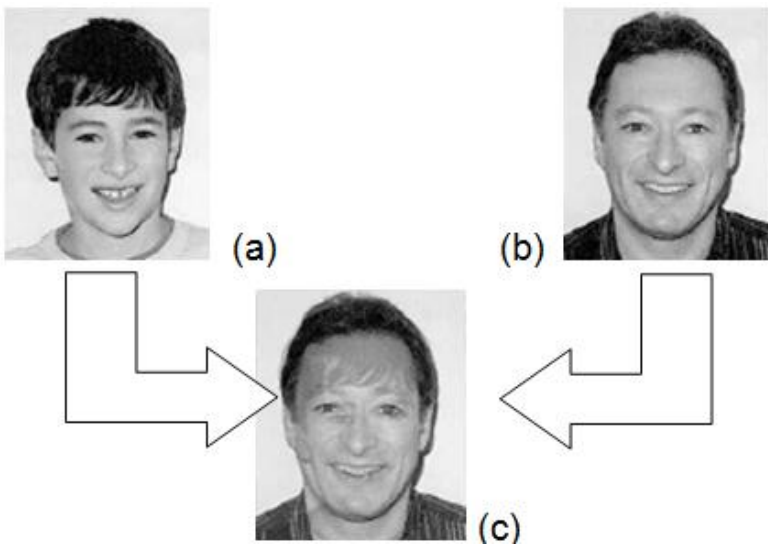

Figura 3. Exemplo de Dissolve.

\section{Warping}

O Warping realiza uma operação de transformação geométrica na imagem modificando a relação espacial de pontos na imagem, podendo realizar translações ou rotações de imagens à deformações no espaço muito complexas. A principal característica da técnica conhecida como Warping é de aumentar e diminuir a imagem através de pontos mapeados no espaço dela através de uma ou várias triangulações, como pode ser visto na imagem abaixo.
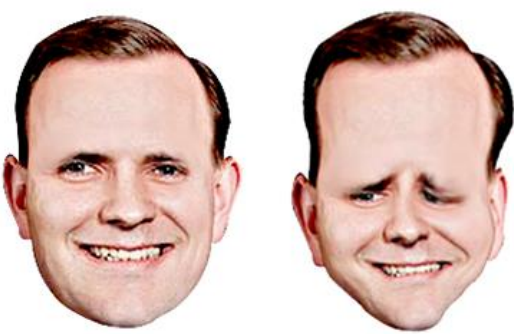

Figura 4. Exemplo de Warping.

Utilizando o Warping, o Morphing vai muito mais além, transformando uma imagem por completo, alterando os valores dos pixels em vez de apenas alterar a relação do espaço entre pontos. Para que isso possa ocorrer é necessário, em ambas as imagens, definir elementos correspondentes, tendo como exemplo a região dos olhos (caso seja uma face). As regiões de interesses podem ser definidas através de pontos, retas, curvas, etc, denominando-se fase de especificação de características.

\section{Triangulação (Mapeamento)}

Os métodos de implementação incluem fazer um mapeamento (Figura 5) em ambas as imagens (original e final), usando pontos correspondentes.
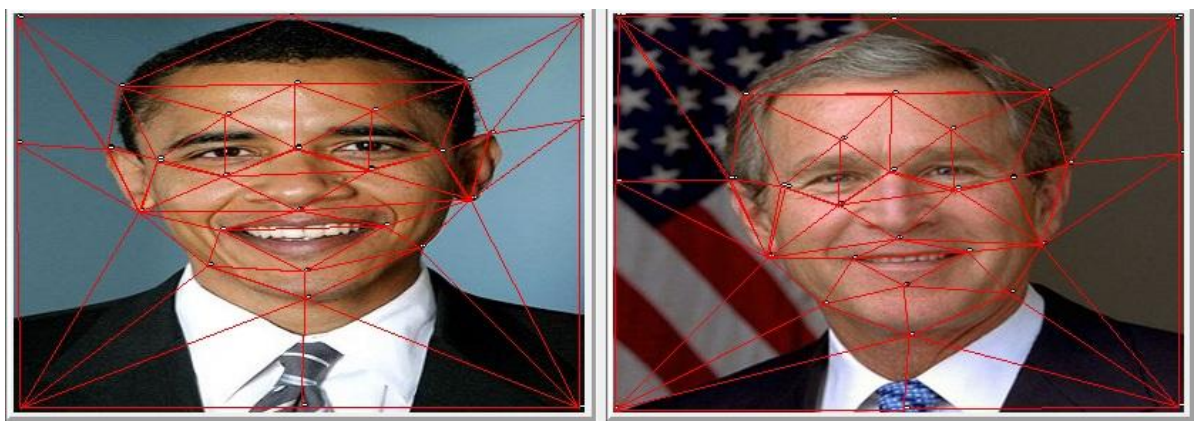

Figura 5. Imagem Mapeada.

Para realizar a triangulação foi utilizado o algoritmo de Bresenham, que tem como objetivo criar o desenho de linhas permitindo saber em quais pixels de uma imagem devem ser destacados para obter o 
grau de inclinação de um ângulo de determinada reta.

\section{Algoritmo Flood-Fill}

O algoritmo de Flood-Fill é responsável pelo percorrer (inundar) os pixels internos de uma área delimitada. Sendo assim é possível utilizar o algoritmo para preencher toda uma área delimitada por uma cor a partir de um ponto inicial. O pseudo-código para o Flood-Fill é apresentado na Caixa 2.

\section{FloodFill (x, y, cor, novaCor)}

Se pixel $(x, y)=$ cor então pixel $(x, y) \leftarrow$ novaCor FloodFill $(x+1, y$, cor , novaCor) FloodFill ( $x, y+1$, cor , novaCor) FloodFill ( $x-1, y$, cor, novaCor) FloodFill ( $x, y-1$, cor , novaCor)

Caixa 2. Pseudo-código para o Fllod-Fill.

\section{Envelhecimento Facial de Imagens}

Nos dias de hoje a Computação Gráfica se encontra aplicada em várias áreas relacionadas da ciência humana, como por exemplo, na área policial:

- Achar fugitivos procurados pela polícia: muitos bandidos não possuem fotos atualizadas, somente de quando eram mais novos, portanto ter um sistema que permita atualizar a aparência física de tais indivíduos permite localizá-los com maior facilidade (CrimeLab 2006).
- Procurar por crianças desaparecidas: aplicação para auxiliar na localização de pessoas desaparecidas por muitos anos, fazendo assim uma simulação de suas características faciais atuais.
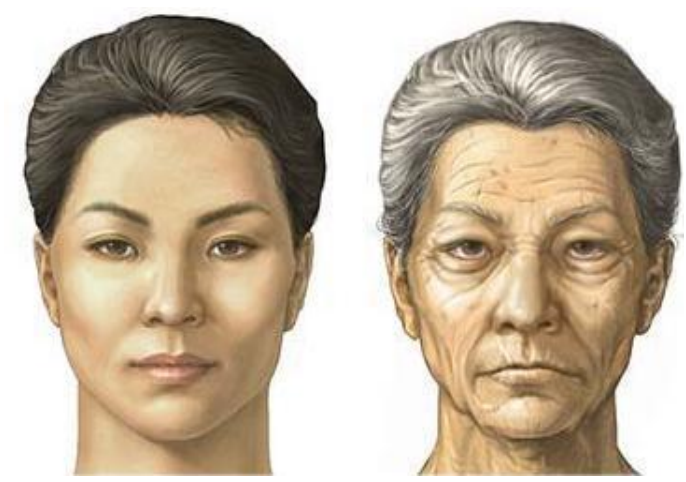

Figura 6. Envelhecimento Facial.

\section{TRABALHO PROPOSTO}

A proposta de trabalho é a construção e implementação de um aplicativo de efeitos especiais que consiste em realizar o Morphing, utilizando o Dissolve, Warping e a Triangulação (Mapeamento) entre duas imagens (Imagem Inicial e Imagem Final), possibilitando a sua continuação em trabalhos de simulação. Após a seleção da Imagem Inicial e Imagem Final é realizado 0 mapeamento dos triângulos correspondentes em ambas às imagens que deverão ser transformados da Imagem Inicial para a Imagem Final (Imagem Inicial $\rightarrow$ Imagem Final).
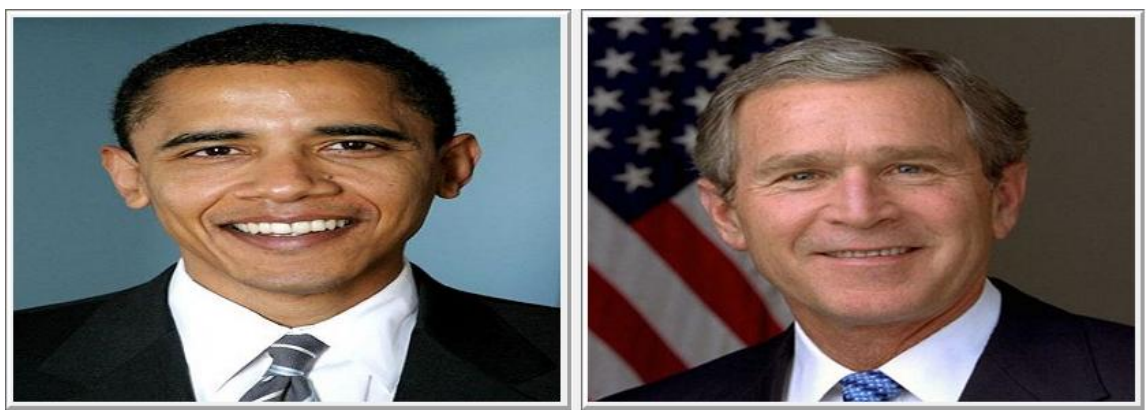

Figura 7. Imagem Inicial e Imagem Final. 


\section{Mapeamento dos Pontos}

Os métodos de implementação incluem fazer um mapeamento em ambas as imagens (Inicial e Final), usando pontos correspondentes nas imagens para a formação dos triângulos, sendo este o caminho a ser percorrido para a aplicação dos efeitos de Morphing, Dissolve e Warping.

\section{Transformação dos Triângulos}

A transformação dos triângulos consiste em obter os vértices de cada triângulo da imagem Inicial, transformando-os nos triângulos da imagem final (Figura 8). Para isso é necessário informar a quantidade de passos $t$ (frames) que irá ocorrer em cada triângulo até que se obtenham todos os triângulos da imagem final.
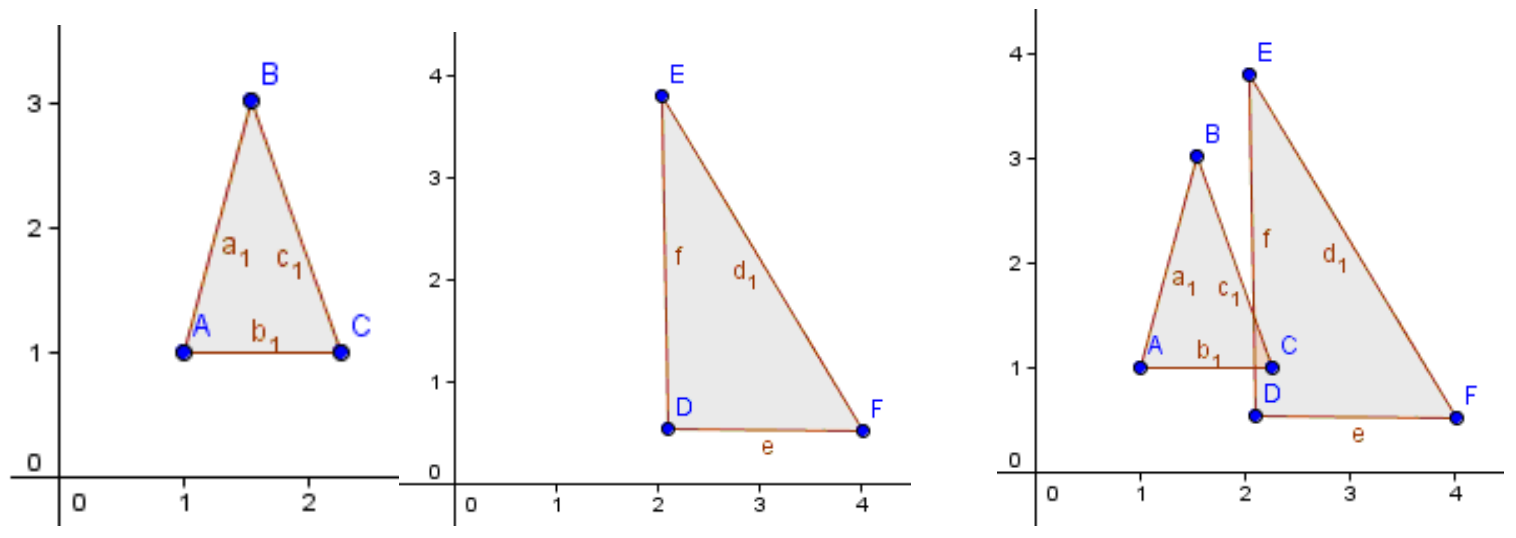

Para obter as movimentações dos triângulos de acordo com o passo atual da operação, utilizase a seguinte Equação 2:

$$
\begin{aligned}
& X=i x+t^{*} v x \\
& Y=i y+t^{*} v y \\
& Z=i z+t^{*} v z
\end{aligned}
$$

Ao obter a movimentação do triângulo que está sendo processado, é calculado o baricentro deste triângulo e a partir do centro, percorre-se todos os pontos internamente com o algoritmo de Flood-Fill, obtendo a interpolação dos pontos internos do triângulo intermediário $t$ no triângulo Inicial da Imagem Inicial e Imagem Final.

Figura 8. Triângulo Inicial e Triângulo Final

Feito a movimentação do triângulo no passo $t$, o próximo passo é percorrer todos os pontos internos deste triângulo para obter sua localização no triângulo que corresponde a Imagem Inicial e Final através da Interpolação para aplicar o efeito Dissolve.

\section{Geração de Frames}

Após realizar o mapeamento dos triângulos informando os pontos equivalentes para que seja possível realizar a transformação de uma imagem em outra, deve-se percorrer os pontos internos de cada triângulo da sequência atual que está sendo processada, mapeando o ponto $\mathrm{x}, \mathrm{y}$ da sequencia $t$ na imagem Inicial e na 
algoritmo Flood-Fill, que é responsável por percorrer todos os pixels internos de cada triângulo da sequência que está sendo processada.

\section{EXPERIMENTOS}

Durante a fase de testes foram realizados vários experimentos, envolvendo diversos tipos de transformações.

\section{Aplicando o Morphing em duas formas geométricas}

A Figura 9 mostra o mapeamento realizado entre um quadrado azul e um pentágono verde. Ao realizar o mapeamento, a fase de transformação que consiste em aplicar os Efeitos de Dissolve e Warping, será responsável para obter as transformações da imagem inicial (quadrado) até a imagem final (pentágono). Abaixo o Efeito de Morphing entre o quadrado e o pentágono.

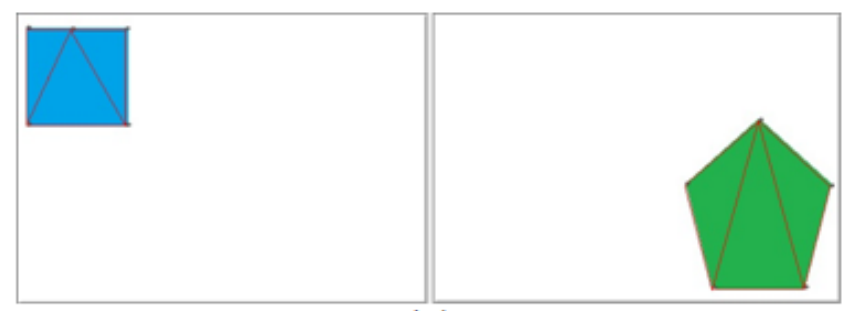

(a)

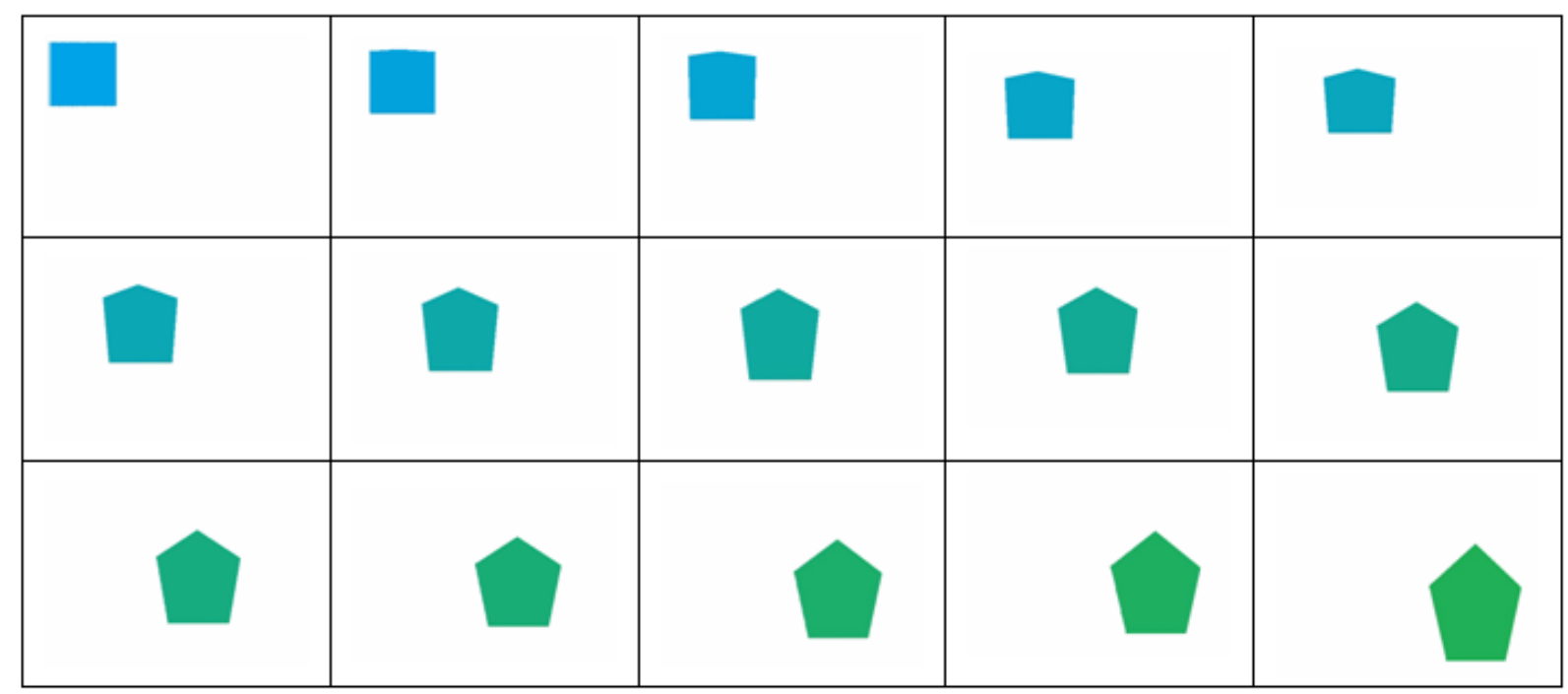

(b)

Figura 9. a) Quadrado azul e retângulo verde, usados para gerar uma b) Sequência de 15 Frames.

\section{Aplicando o Morphing em duas faces}

A Figura 10 mostra um mapeamento proposto para as duas imagens, usadas para gerar os frames apresentados na Figura 11. 

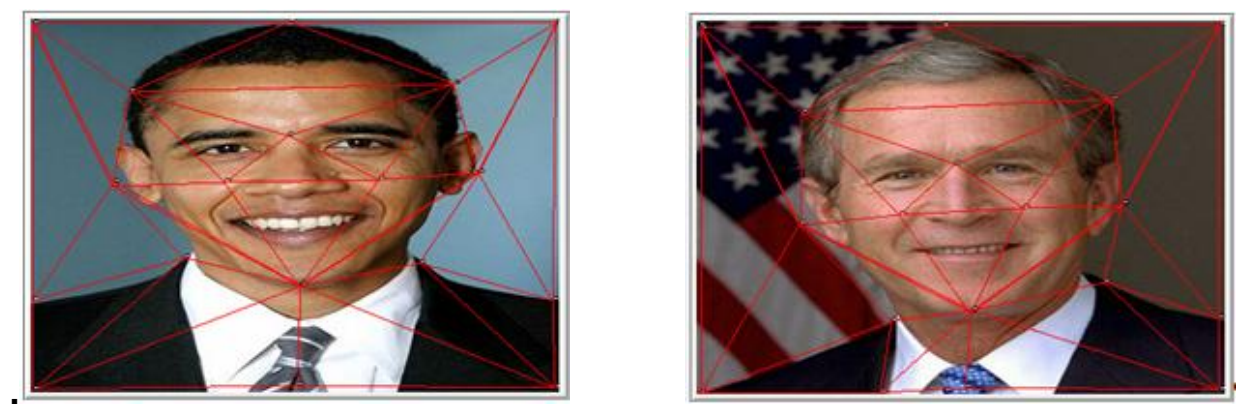

Figura 10. Mapeamento entre faces

A transformação do mapeamento, como mostra na Figura 11, foi realizada com $t=30$ (trinta passos). Abaixo, o resultado passo-a-passo desta transformação.

\section{CONSIDERAÇÕES FINAIS}

Para realizar a implementação do aplicativo foi necessário buscar conhecimentos nos conceitos de Dissolve e Warping para que fosse possível realizar a fusão dessas duas técnicas e

Durante a execução das técnicas implementadas no aplicativo, foi constatado que quanto maior a quantidade de triângulos para realizar as transformações geométricas de Warping no espaço da imagem e também um número maior de frames para realizar a sequência do Dissolve, maior é a carga de processamento, fazendo com que a operação de Morphing se torne muito demorada. obter a técnica de Morphing.

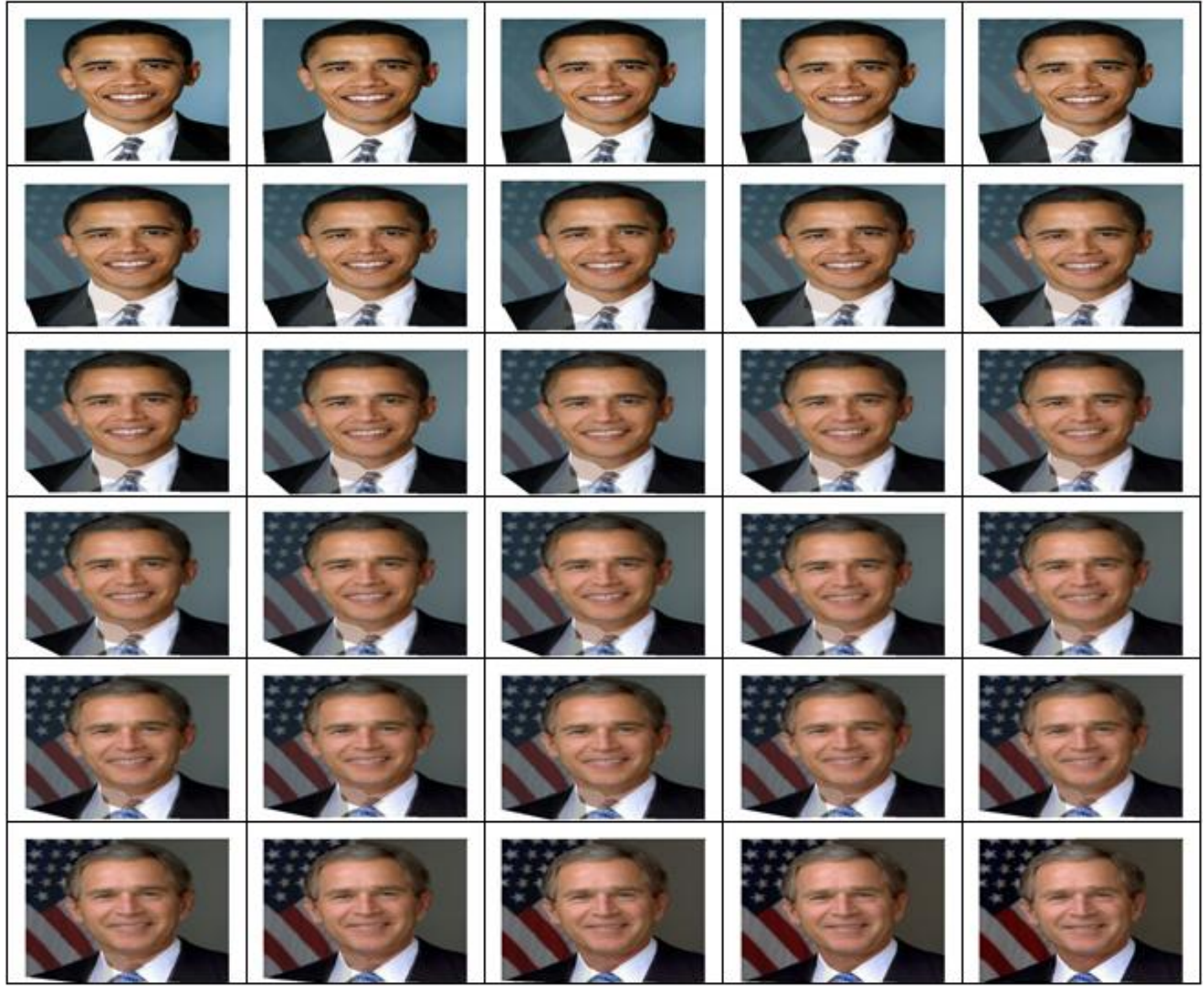

Figura 11. Sequência com 30 Frames mostrando a transformação das imagens na Figura 10. 


\section{REFERÊNCIAS}

FOLEY, James D.; VAN DAM, Andreies, FEINER, Steven K., GORDON, Peter S., C\&C Associates, Computer grafics: principles and practice. Addison-Wesley, 1997.

GOMES, Jonas; VELHO, Luiz; Computação Gráfica: Imagens, Rio de Janeiro: IMPA/SBM, 1994.

GONZALES, Rafael C.;Woods, Richard E.; Processamento de Imagens Digitais,São Paulo: Edgard Blucher , 2000.

HEARN, Donald, BAKER, M. Paulin; Computer graphics: C version. Prentice Hall, 1997.

SCHROEDER, Greyce Nogueira; Morphing aplicado ao envelhecimento de imagens Faciais. Campinas/SP,2007.

STEINBRUCH, Alfredo; WINTERLE, Paulo; Geometria Analítica. São Paulo, Pearson Makron Books,1987. 